\title{
TP53 Gene 72 Arg/Pro (rs1042522) Single Nucleotide Polymorphism Contribute to Increase the Risk of B-Chronic Lymphocytic Leukemia in the Sudanese Population
}

\author{
Ameen Abdulaziz Mohammed Basabaeen 1,2, Enaam Abdalrhman Abdelgader ${ }^{3}$, \\ Ebtihal Ahmed Babekir ${ }^{1}$, Saadia Osman Abdelrahim ${ }^{1}$, Nada Hassan Eltayeb ${ }^{4}$, \\ Osama Ali Altayeb ${ }^{5}$, Eman Abbass Fadul ${ }^{5}$, Abdulwali Sabo ${ }^{6}$, Ibrahim Khider \\ Ibrahim $^{1,7 *}$
}

\begin{abstract}
Objective: This study aimed at exploring the association of TP53 $72 \mathrm{Arg} /$ Pro polymorphism and Risk of Chronic Lymphocytic Leukemia and to assess the correlation between TP53 72Arg/Pro polymorphism and clinical parameter, hematological profile and some biological prognostic markers among Sudanese patients with chronic lymphocytic leukemia. Methods: A case-control study was conducted in Khartoum state, Sudan, during the period from April 2017 to April 2018, involved 110 B-CLL patients and 80 healthy volunteers as a control group. Physical examination, Complete Blood Count and Immunophenotype were performed in all patients to confirm the diagnosis. Clinical staging such as Rai and Binet were studied. CD38 and ZAP70 were performed by Flow Cytometry. Blood samples were collected from all participants; DNA was extracted by using ANALYTIKJENA Blood DNA Extraction Kit (Germany) and analyzed TP53 codon 72Arg/Pro Polymorphism by using AS-PCR. The statistical analysis was performed using SPSS version 23.0 software (Chicago, IL, USA). Results: the Arg/Pro was the most frequent genotype in B-CLL patients $(50 \%)$, followed by Arg/Arg (25.5\%) and Pro/Pro (24.5\%), whereas in healthy control group Arg/Pro was the most frequent (47.5\%), followed by $\mathrm{Arg} / \mathrm{Arg}$ (45\%) and Pro/Pro (7.5\%). Our data indicate a higher frequency of homozygous Pro/ Pro in the B-CLL patients as compared to controls with an OR of 4.01 for the Pro/Pro genotype and lower frequency of Arg/Arg genotype in CLL patients as compared to controls with an OR of .42 for the Arg/Arg genotype. Also, the Pro allele showed higher risk than Arg allele ( $\mathrm{P}$ value $=0.000$, OR 2.23, 95\% CI $=1.45-3.41$ ). No significant association between gender, clinical staging systems (Rai, Binet), biological prognostic markers (CD38 expression or ZAP70 expression), and TP53 codon 72Arg/Pro polymorphisms, except Arg/Arg genotype tended to be associated with younger age $(\mathrm{P}=0.04)$. Conclusion: Our data suggested that Pro/Pro genotype contribute to increased susceptibility to B-Chronic Lymphocytic Leukemia risk in our population tenfold higher than those had Arg/Arg genotype.
\end{abstract}

Keywords: CLL- TP53 gene- SNP- 72 Arg/Pro Polymorphism- Risk- Sudan

Asian Pac J Cancer Prev, 20 (5), 1579-1585

\section{Introduction}

B cell chronic lymphocytic leukemia (CLL) is the most common type of leukemia in adults in Western countries, and mainly affects elderly individuals (Chiorazzi et al., 2005). It is a malignancy of mature clonal B lymphocytes that accumulate in the blood, bone marrow and other lymphoid tissues, and the presence of $\geq 5,000$ clonal $\mathrm{B}$ lymphocytes per microliter of peripheral blood persisting for more than 3 months considered positive (Hallek et al., 2008; Hallek, 2017). TP53 play a critical role in regulating tumor growth and survival in CLL demonstrated by the fact that mutational inactivation of this tumor suppressor is associated with aggressive disease and poor prognosis (Dohner et al., 1995; Cordone et al., 1998). TP53 plays a pivotal role in response to genotoxic insults from endogenous or environmental agents by orchestrating a diversity of pathways from activation of cell signaling transduction, transcriptional responses, DNA repair to regulation of cell cycle progression and apoptosis (Hainaut and Wiman, 2009).

The TP53 is encoded by the TP53 gene, which

${ }^{1}$ Department of Haematology, Faculty of Medical Laboratory Sciences, ${ }^{3}$ Department of Pathology, ${ }^{4}$ Department of Physiology Faculty of Medicine, Al Neelain University, ${ }^{5}$ Flow Cytometry Laboratory for Leukemia and Lymphoma Diagnosis Khartoum, Sudan, ${ }^{2}$ Ministry of Health, Hadhramout, Yemen, ${ }^{6}$ Department of Statistics, ${ }^{7}$ Department of Hematology, School of Medical Sciences, University Sains Malaysia, Health Campus, Kubang Kerian, Kelantan, Malaysia.*For Correspondence: ibrahimkh82@gmail.com 
located on chromosome 17q13 (Isobe et al., 1986). Several polymorphisms (over 200 single nucleotide polymorphisms SNPs) have been identified within TP53 gene, both in non-coding and coding regions (Olivier et al., 2002). One of most common SNPs of the TP53 gene is 72 Arg/Pro (rs 1042522). The TP53 codon 72 polymorphism is located in exon 4 with CGC to CCC transition, leading to an Arginine-to-Proline amino acid substitution in amino acid position 72 (Whibley et al., 2009). These SNP appear to be different both biochemically and biologically (Thomas et al., 1999; Dumont et al., 2003; Pim and Banks, 2004).The laboratory studies demonstrated that Arg variant is more potent in apoptosis induction whereas the Pro variant is better in inducing cell cycle arrest and DNA damage repair (Dumont et al., 2003; Pim and Banks, 2004; Siddique and Sabapathy, 2006). The polymorphism is balanced, varies with latitude and race, and is maintained at different allelic frequencies across the population (Själander et al., 1995). Kochethu et al., 2006 (Kochethu et al., 2006) found no significant effect of this polymorphism on CLL biology, except for a weak association between $\mathrm{Arg} / \mathrm{Arg}$ homozygosity and CD38 negativity ( $\mathrm{P}=0.049$ ), while, (Sturm et al., 2005) found no association with Binet stage, IgHV status, and TP53 mutational status, OS or in vitro drug sensitivity. Recent study shown that the Proline allele in the homozygous state was associated with a shorter time to the first treatment among the group of patients with mutated IGHV locus (Majid et al., 2011).

To the best of our knowledge this is the first study with large sample size conducted in Sudan to investigate the association between the TP53 Arg/Pro polymorphism and CLL and correlate with clinical and Hematological parameters. This present study was conducted to examine the association between the P53 Arg 72 Pro polymorphism and Risk of Chronic Lymphocytic Leukemia and to assess correlation between P53 Arg72Pro polymorphism and clinical parameter, hematological profile and some biological prognostic markers among Sudanese patients with chronic lymphocytic leukemia.

\section{Material and Methods}

\section{Study Population}

This study is a case-control study, conducted in Khartoum state, Sudan, in the period from April 2017 to April 2018, a total of 110 patients with Chronic Lymphocytic Leukemia and 80 apparently healthy volunteers as a control group were recruited to participate in this study. Patients were obtained at Flow Cytometry Laboratory for Leukemia \& Lymphoma Diagnosis, Khartoum; they were referred for Immunophenotype diagnosis.

All patients were diagnosed based on clinical history, physical examination and complete blood count. The peripheral blood is important to show morphological abnormalities and immunophenotypic criteria. However, B lymphocyte $\geq 5,000 \times 109 / 1$, considered as a positive in our diagnosis according to International Workshop on Chronic Lymphocytic Leukemia (Hallek et al., 2008). The stage of the Chronic Lymphocytic Leukemia was assessed by Rai et al., (1975) and Binet et al., (1981) classification. All patients were newly diagnosed without any previous treatment, whereas patients with other lymphoid neoplasms (both B and T-cell Lineage) were excluded.

Determination of Blood Count, Immunophenotyping and (CD38 and ZAP-70 expression).

Two $\mathrm{ml}$ of peripheral blood (PB) were withdrawn from each patient; these samples were collected in EDTA tubes and preserved at room temperature $\left(22-24^{\circ} \mathrm{C}\right)$ then processed within $6-24 \mathrm{~h}$ from the collection. Complete blood count was analyzed by using automated hematology analyzer (Sysmmex XE-2100TM, Kobe Japan). All results such total WBC, Absolute lymphocyte count, Hemoglobin level, RBC and platelets were recorded. And a blood smear stained by May Grunwald Giemsa was obtained for all patients

The diagnosis of CLL was confirmed in each patient by Flowcytometry (EPICS XL Beckman Coulter Flow Cytometry, Miami, FL, USA), standard protocol of Beckman Coulter was used in fluorescent dye-labelled monoclonal antibody for CD45, CD3 CD5, CD10, CD19, CD20, CD22, CD23, FMC7, CD79b, kappa and lambda light chain. A marker was considered positive at cutoff $\geq 30 \% .30 \%$ cutoff was selected as recommended by British Committee for Standards in Haematology (BCSH) guideline (Oscier et al., 2012). However, in order to confirm diagnostic CLL, a scoring system was applied depending on (Moreau et al., 1997), this scoring system allocated one point for each following markers expressed $\mathrm{CD} 5$ and CD23 while absence or low expression of SmIg, CD79b and FMC7. Absolute B lymphocyte count was obtained by Flowcytometry and ZAP-70 and CD38 were used as prognostic markers, with a cutoff point of $20 \%$ and $30 \%$, respectively.

\section{DNA extraction}

After confirmed immunophenotyping of patients, genomic DNA was extracted from all blood samples of patients and control groups by using ANALYTIKJENA Blood DNA Extraction Kit (Germany) (REF-845-KS-1020050), according to the manufacturer's instructions. The $\beta$-globin gene was used to assess the quality of DNA in all extracted samples, $\beta$ globinGH20 (5'-GAAGAGCCAAGGACAGGTAC-3') and $\beta$ globin-PC04 (5'-CAACTTCATCCACGTTCACC-3') as previously described (Kerr et al., 2000). All specimens for $\beta$-globin gene were Successful amplification with product size 268bp. To evaluate the DNA quantification after DNA extraction, we measured DNA by using a NanoDrop spectrophotometer. Then DNA samples were routinely stored at $-20^{\circ} \mathrm{C}$.

Genotyping of TP53 exon 4 codon 72 Arg/Pro (rs 1042522) SNP (rs 1042522) TP53 gene was performed by Allele-Specific Polymerase Chain Reaction (AS-PCR), the following primers were used, Proline: Forward (5'-GCCAGAGGCTGCTCCCCC-3'), reverse (5'-CGTGCAAGTCACAGACTT-3'); Arginine: Forward (5'-TCCCCCTTGCCGTCCCAA-3'), reverse 
(5'-CTGGTGCAGGGGCCACGC-3'), The PCR products were $177 \mathrm{bp}$ and $141 \mathrm{bp}$ for Proline and Arginine, respectively, as previously reported (Bereir et al., 2003). PCR products and 50 bp DNA ladder (iNtRON BIOTECHNOLOGY, KOREA), were run on $2 \%$ agarose gel containing ethidium bromide and identified under UV transilluminator using gel documentation system (SYNGENE, JAPAN).

\section{Statistical analysis}

Patient's data was collected by structural interview questionnaire and from patient's medical files and analyzed using the statistical package for social sciences (SPSS) version 23.0 software (Chicago, IL, USA). Numerical data was summarized as mean and stander deviation. Chi Square test was used for analyzing qualitative data. Logistic regression was used for calculation of odds ratio (OR) with confidence interval (CI) for risk estimation. The Hardy-Weinberg equilibrium was tested by goodness of fit $\mathrm{X}^{2}$ test to compare the observed genotypic frequencies in normal individual to the expected genotypic frequencies, and then calculated from the observed allelic frequencies. A P-value $<0.05$ was considered significance.

\section{Results}

In this study, 110 cases of CLL were studied, the overall mean age was $(62.97 \pm 12.061)$ with range $(22$ $85 y)$. Seventy-nine $(71.8 \%)$ were males while 31 (28.2\%) were females; Male to female ratio was $(2.6: 1)$. The patients classified according to Rai staging system, 9.1\% stage $0,20.9 \%$ stage I, $20.9 \%$ stage II, $32.7 \%$ stage III, and $16.4 \%$ stage IV. While, Binet stage distribution was: stage A, $30.0 \%$; stage B, $31.8 \%$ and stage C, $38.2 \%$. Mean white blood cell count was $92.86 \pm 75.43 \times 10^{3} / \mathrm{ul}$, mean platelet count was $189.24 \pm 104.91 \times 10^{6} / \mathrm{ul}$ and Mean hemoglobin level was $11.2 \pm 2.5 \mathrm{~g} / \mathrm{dL}$. Flow cytometric analysis revealed CD38 expressed in $37.7 \%$ of patients and ZAP70 expressed in $32.7 \%$ of patients.

Associations between TP53 codon 72Arg/Pro genotypes and alleles and B-CLL risk

One picture of gel electrophoresis explained different genotypes of TP53 Arg/Pro are shown in Figure 1. The frequencies of TP53 genotype were determined by AS-PCR in 110 CLL patients and 80 (age and sex-matched) healthy volunteers were enrolled as control

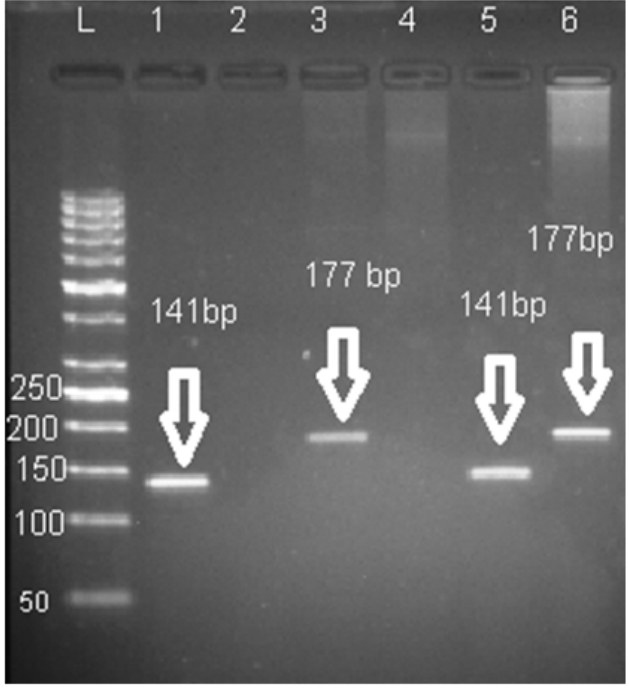

Figure 1. It Shows the 2 Sized Amplicons Outcome of AS-PCR. Well L indicates the ladder of $50 \mathrm{bp}$.Amplicon $141 \mathrm{bp}$ indicates the presence of Arg allele, Amplicon of size $177 \mathrm{bp}$ indicates the presence of Pro allele. Wells 1, 2 indicates the homozygous Arg/Arg genotype. Wells: 3, 4 indicates the homozygous Pro/Pro genotype. Wells: 5, 6 indicates the heterozygous Arg/Pro genotype.

group. The Arg/Pro was the most frequent genotype in patients with CLL (50\%), followed by Arg/Arg (25.5\%) and Pro/Pro (24.5\%) whereas in healthy control group Arg/Pro was the most frequent (47.5\%), followed by Arg/ Arg (45\%) and Pro/Pro (7.5\%), (See Figure 2 ). these frequencies were consistent with the Hardy-Weinberg equilibrium (HWE). In our patient's samples, the allelic frequencies of Arg and Pro were 0.50 while in control samples were 0.69 and 0.31, respectively, (See Table 1 ).

The Pro/Pro genotype of TP53 SNP showed higher risk of B-CLL compared with Arg/Arg; which was statistically significant ( $\mathrm{P}$ value $=0.002$, OR 4.01, 95\% CI=1.57-10.26). Not surprising, the risk was lower when adding Arg/Pro + Pro/Pro in patients compared to $\mathrm{Arg} / \mathrm{Arg}$ ( $\mathrm{P}$ value $=0.004$, OR 2.4, 95\% $\mathrm{CI}=1.3-4.43$ and $\mathrm{P}$ value $=0.004$, OR 0.42, 95\% CI=0.23-0.77, respectively), See Table 1 ).

Not only TP53 codon 72 genotypes but also TP53 allele's frequencies were studied. Arg and Pro allele's frequencies of TP53 codon 72 SNP in CLL patients were $110(50 \%)$. Compared to these alleles in the control group, $110.4(69 \%)$ and $49.6(31 \%)$, respectively. The Pro allele showed higher risk compared with Arg allele

Table 1. Frequency of the TP53 Codon 72 Arg/Pro Genotype in Patients with Chronic Lymphocytic Leukemia and Control Group:

\begin{tabular}{lcccc}
\hline Allele / Genotype & CLL group (N=110) (\%) & Control group (N=80) (\%) & Odds ratio (95\% CI) & P-value \\
\hline Arg/Arg & $28 / 110(25.5 \%)$ & $36 / 80(45 \%)$ & $0.42(0.23-0.77)$ & 0.004 \\
Arg/Pro & $55 / 110(50 \%)$ & $38 / 80(47.5 \%)$ & $1.11(0.62-1.97)$ & 0.733 \\
Pro/Pro & $27 / 110(24.5 \%)$ & $6 / 80(7.5 \%)$ & $4.01(1.57-10.26)$ & 0.002 \\
Arg allele frequency & $110(50 \%)$ & $110.4(69 \%)$ & $0.45(0.29-0.69)$ & 0 \\
Pro allele frequency & $110(50 \%)$ & $49.6(31 \%)$ & $2.23(1.45-3.41)$ & 0 \\
Arg/Pro+Pro/Pro & $82 / 110(74.5)$ & $44 / 80(55 \%)$ & $2.4(1.3-4.43)$ & 0.004 \\
Arg/Arg & $28 / 110(25.5 \%)$ & $36 / 80(45 \%)$ & $0.42(0.23-0.77)$ & 0.004 \\
\hline
\end{tabular}

Chi Square test, $(\mathrm{n}=110), \mathrm{P}$ value significant below 0.05 . Arg/Pro 
Table 2. Associations between TP53 Codon 72 Arg/Pro Genotype and CLL Prognostic Markers

\begin{tabular}{|c|c|c|c|c|}
\hline \multirow[t]{2}{*}{ Prognostic marker } & \multicolumn{3}{|c|}{ Genotype } & \multirow[t]{2}{*}{ P- value } \\
\hline & $\mathrm{Arg} / \mathrm{Arg}$ & Arg/Pro & Pro/Pro & \\
\hline No. of Cases & 28 & 55 & 27 & $0.001^{\mathrm{a}}$ \\
\hline HWE & 28 & 55 & 27 & \\
\hline Mean Age & $58.82 y$ & $63.09 y$ & $67.04 y$ & $0.040^{\mathrm{b}}$ \\
\hline \multicolumn{5}{|l|}{ Sex } \\
\hline Male & $18 / 79(22.8 \%)$ & $39 / 79(49.4 \%)$ & $22 / 79(27.8 \%)$ & $0.358^{\mathrm{c}}$ \\
\hline Female & $10 / 31(32.3 \%)$ & $16 / 31(51.6 \%)$ & $5 / 31(16.1 \%)$ & \\
\hline \multicolumn{5}{|l|}{ Binet Stage } \\
\hline A & 9/33 (27.3\%) & $18 / 33(54.5 \%)$ & $6 / 33(18.2 \%)$ & \\
\hline $\mathrm{B}$ & $11 / 35(31.4 \%)$ & $16 / 35(45.7 \%)$ & $8 / 35(22.9 \%)$ & $0.598^{c}$ \\
\hline $\mathrm{C}$ & $8 / 42(19.0 \%)$ & $21 / 42(50.0 \%)$ & $13 / 42(31.0 \%)$ & \\
\hline \multicolumn{5}{|l|}{ Rai Stage } \\
\hline 0 & $4 / 10(40.0 \%)$ & $4 / 10(40.0 \%)$ & $2 / 10(20.0 \%)$ & \\
\hline $\mathrm{I}$ & $8 / 23(34.8 \%)$ & 9/23 (39.1\%) & $6 / 23(26.1 \%)$ & \\
\hline II & $6 / 23(26.1 \%)$ & $13 / 23(56.5 \%)$ & 4/23 (17.4\%) & $0.158^{\mathrm{c}}$ \\
\hline III & $4 / 36(11.1 \%)$ & $24 / 36(66.7 \%)$ & $8 / 36(22.2 \%)$ & \\
\hline IV & 6/18 (33.3\%) & $5 / 18(27.8 \%)$ & $7 / 18(38.9 \%)$ & \\
\hline \multicolumn{5}{|l|}{ CD38 } \\
\hline$<30 \%$ & $19 / 69(27.5 \%)$ & $33 / 69(47.8 \%)$ & $17 / 69(24.6 \%)$ & $0.782^{\mathrm{c}}$ \\
\hline$\geq 30 \%$ & 9/41 (22.0\%) & $22 / 41(53.7 \%)$ & $10 / 41(24.4 \%)$ & \\
\hline \multicolumn{5}{|l|}{ ZAP70 } \\
\hline$<20 \%$ & $22 / 74(29.7 \%)$ & $35 / 74(47.3 \%)$ & $17 / 74(23.0 \%)$ & $0.336^{\mathrm{c}}$ \\
\hline$\geq 20 \%$ & $6 / 36(16.7 \%)$ & $20 / 36(55.6 \%)$ & $10 / 36(27.8 \%)$ & \\
\hline
\end{tabular}

${ }^{\mathrm{a}}$, compared to the control group; ${ }^{\mathrm{b}}$, ANOVA test; ${ }^{\mathrm{c}}$, Chi Square test; $(\mathrm{n}=110), \mathrm{P}$ value significant below 0.05 .

( $\mathrm{P}$ value $=0.000, \mathrm{OR} 2.23,95 \% \mathrm{CI}=1.45-3.41$ and $\mathrm{P}$ value $=0.000$, OR $0.45,95 \% \mathrm{CI}=0.29-0.69$, respectively), (See Table 1 ).

Associations between TP53 codon 72 Arg/Pro and clinical parameters and biological prognostic markers

TP53 codon 72 genotype differed significantly in median age of diagnosis between the three groups; 58.8, 63 and 67 years in patients with Arg/Arg, Arg/Pro and Pro/Pro genotypes, respectively. (P value=0.04; (Data was shown in Table 2 ).
In the current study, there is no significant association between gender, clinical staging systems (Rai, Binet), biological prognostic markers (CD38 expression or ZAP70 expression), and TP53 codon 72 polymorphisms. (All $\mathrm{P}$ value $>0.05$; Data was shown in Table 2 ).

Associations between TP53 codon 72 Arg/Pro and hematological parameters

The results showed that no statistically significant difference was found in total white blood cell count, differential white count, Platelet count, red blood

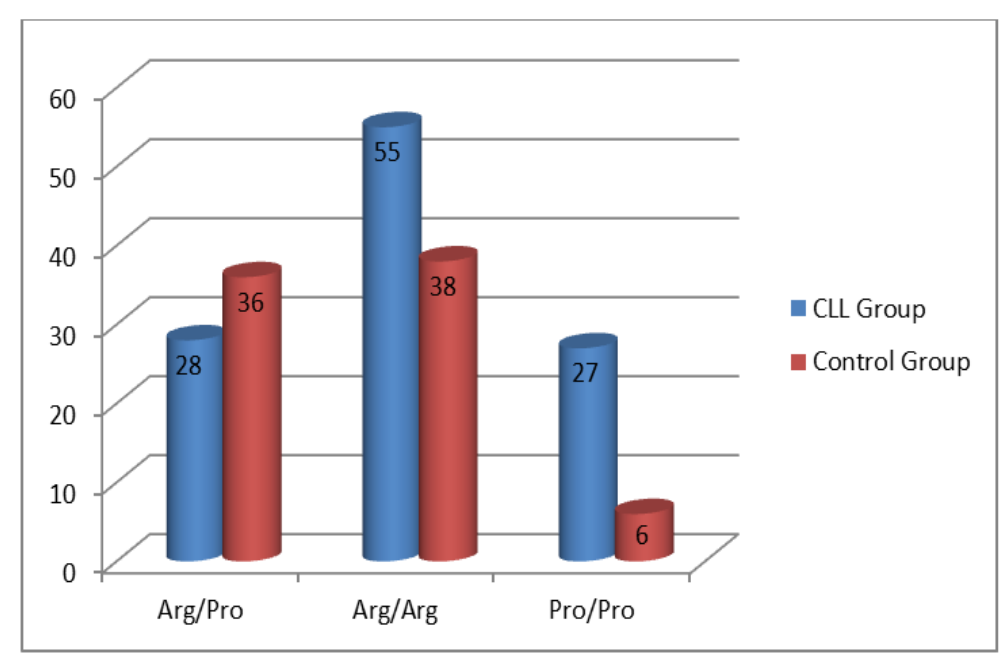

Figure 2. Comparison of Genetic Distribution in Patients with Chronic Lymphocytic Leukemia and Control Group 
DOI:10.31557/APJCP.2019.20.5.1579

TP53 Gene 72 Arg/Pro (rs1042522) Single Nucleotide Polymorphism Contribute

Table 3. Associations between TP53 Codon 72 Arg/Pro Genotype and Hematological Parameters

\begin{tabular}{|c|c|c|c|c|c|c|c|}
\hline \multirow[t]{4}{*}{ Parameter } & \multicolumn{6}{|c|}{ Genotype } & \multirow[t]{4}{*}{ P. value } \\
\hline & \multirow{2}{*}{\multicolumn{2}{|c|}{$\begin{array}{c}\text { Arg/Arg } \\
\text { No. } 28\end{array}$}} & \multirow{2}{*}{\multicolumn{2}{|c|}{$\begin{array}{c}\text { Arg/Pro } \\
\text { No.55 }\end{array}$}} & \multirow{2}{*}{\multicolumn{2}{|c|}{$\begin{array}{c}\text { Pro/Pro } \\
\text { No. } 27\end{array}$}} & \\
\hline & & & & & & & \\
\hline & Mean & SD & Mean & SD & Mean & SD & \\
\hline $\mathrm{WBC} \times 10^{3} / \mathrm{ul}$ & 85.45 & 68 & 98.29 & 85.17 & 86.72 & 63.6 & 0.7 \\
\hline Absolute- Lymphocyte $\times 10^{3} / \mathrm{ul}$ & 78.46 & 73.53 & 84.23 & 74.17 & 82.07 & 63.21 & 0.94 \\
\hline Absolute B Lymphocyte $\times 10^{3} / \mathrm{ul}$ & 69.93 & 72.29 & 74.75 & 68.32 & 73.92 & 60.03 & 0.95 \\
\hline $\mathrm{RBC} \times 10^{6} / \mathrm{ul}$ & 3.87 & 0.94 & 3.66 & 0.99 & 3.79 & 1.04 & 0.65 \\
\hline Platelets $\times 10^{3} / \mathrm{ul}$ & 199.57 & 100.82 & 184.43 & 96.75 & 171.19 & 76.53 & 0.53 \\
\hline Hemoglobin (g/dl) & 11.67 & 2.22 & 11.07 & 2.63 & 11.37 & 2.85 & 0.6 \\
\hline Granulocytes \% & 12.36 & 7.55 & 13.65 & 7.67 & 11.7 & 7.73 & 0.51 \\
\hline Monocytes \% & 2.04 & 1.42 & 2.87 & 1.98 & 2.81 & 2.35 & 0.16 \\
\hline Lymphocyte \% & 85.61 & 8.01 & 83.47 & 8.9 & 85.48 & 9.39 & 0.46 \\
\hline
\end{tabular}

ANOVA test; $(\mathrm{n}=110)$. P value significant below 0.05

cell count, hemoglobin level and TP53 codon 72 polymorphisms. (All $\mathrm{P}$ value $>0.05$; Data was shown in (Table 3).

\section{Discussion}

CLL heterogeneity may be associated with single nucleotide polymorphic (SNP) variation. In the recent years a simple single nucleotide polymorphism arrays proved to be efficient techniques to detect genetic variation in malignant cell, including CLL (Maciejewski et al., 2009). A sequence polymorphism at codon 72 of the TP53 gene (exon 4) encoding either Arg (CGC) or Pro has been suggested to drastically alter the biological and biochemical behavior of TP53 in vitro (Sturm et al., 2005).

The present study revealed that TP53 polymorphism $72 \mathrm{Arg} /$ Pro (rs 1042522) plays a significant role in B-CLL risk in our population. This was demonstrated by analyzing the SNP by AS-PCR approach individually and in combination, where the frequency of Pro allele and the homozygous Pro/Pro genotype were highly increased in patients with B-CLL and higher risk of developing B-CLL (Odds Ratio for variant Pro allele and for Pro/Pro genotypes, 2.23 and 4.01 , respectively.

Our data indicate a higher frequency of homozygous Pro/Pro in the B-CLL patients as compared to controls with an OR of 4.01 for the Pro/Pro genotype and lower frequency of $\mathrm{Arg} / \mathrm{Arg}$ genotype in CLL patients as compared to controls with an OR of .42 for the Arg/ Arg genotype. In other words, patients with the Pro/Pro genotype increase susceptibility to B-CLL tenfold higher than those had Arg/Arg genotype.

Our result shows that the codon 72 Pro/Pro genotype is more predominance in CLL patients compared to our control and suggested that this genotype predisposes to the development of CLL which is contradict with previous study that found the Arg/Arg genotype most common genotype in patients with Chronic Lymphocytic Leukemia and suggested that this genotype predisposes to the development of CLL (Kochethu et al., 2006). Our result was consistent with recently study by Dong et al., (2014) suggested that the Pro allele may predispose individuals to the development of CLL.

A meta-analysis of thirteen case-control studies showed that there is no significant association was found between this polymorphism and leukemia risk (Weng et al., 2012). Also more recently another meta-analysis found that there is no statically significant risk of TP53 Arg/Pro polymorphism in leukemia and no significant association in a subgroup analysis by ethnicity except Pro/Pro genotype may increase the risk of ALL (Tian et al., 2016). The relationship between TP53 $72 \mathrm{Arg} / \mathrm{PrO}$ polymorphism and risk of leukemia remains controversial.

In our study, we found that there was significantly difference in allele $(\mathrm{P}=0.0003)$ and genotype $(\mathrm{P}=0.001)$ distributions of $72 \mathrm{Arg} / \operatorname{Pro}$ (rs 1042522) among CLL patients and controls. In contrast to our study, The TP53 $72 \mathrm{Arg} /$ Pro polymorphism in the current series of CLL patients displayed similar frequencies to those in previously examined healthy control from Sudanese population (Bereir et al., 2003). This may be due to small size of our control group; difference in ethnic populations from Sudan indicated significant intra-population differences in allele frequency and genotype distribution.

Many studies showed that the frequency of TP53 Arg/ Pro polymorphism variants differ among populations from various region in world. In Northern Europeans the Pro72 haplotype is present in about $10 \%$ of the population but in Nigeria it is found in 63\% (Själander et al., 1995) .

Previous report in Sudan conducted by (Mohand Altayeb Mohamed et al., 2013) found association between Arg/Pro genotype and the risk of both ALL and CLL, but their patients sample size was only fifteen patients with chronic lymphatic leukemia. Our study shows that the Arg/Pro most common genotype than Arg/Arg or Pro/Pro in CLL patients but the difference was not statistically significant, this disagrees with previous report by Kochethu et al., (2006) and Lahiri et al., (2007), both studies in UK showed higher frequency of Arg/Arg than Arg/Pro or Pro/Pro in CLL patients, also other study by Sturm et al., (2005) in Germany revealed that same result. In agreement with our findings, Dong et al., (2014) in China find $47.3 \%$ was heterozygous (Arg/Pro) while $29.6 \%$ and $21.3 \%$ homozygous (Arg/Arg) and (Pro/Pro), 
respectively.

Pro/Pro genotype was more predominant in our patients than above studies from the UK (Kochethu et al., 2006; Lahiri et al., 2007) and Germany (Sturm et al., 2005). These findings were consistent with (Dong et al., 2014). The reasons for the discrepancies between different studies are not clear. However, some explanations can be suggested including that the differences may be due to variable geographical distribution and also some interethnic differences may be involved.

Association between TP53 Arg/Pro and clinical, biological markers in CLL previous reports were contradictory findings, while a recent study has shown the Proline allele in the homozygous state was associated with a shorter time to the first treatment among the group of patients with mutated IGHV locus (Majid et al., 2011). Also, Kochethu et al., (2006) found that the only weak association between Arg/Arg and CD38 negativity ( $\mathrm{P}=0.049)$. Dong et al., (2014) found that significant association between Pro/Pro genotype and TP53 deletion, mutations. Bilous et al., (2014) suggested that CLL patients with the Pro/Pro genotype are risk group for the development of TP53 mutations. On the other side Sturm et al., (2005), Lahiri et al., (2007) and Dong et al., (2014) found that there is no significant association between TP53 Arg/Pro polymorphism and other parameters such as age, sex, Binet stage, CD38 expression, and ZAP70 expression.

In our study found that $\mathrm{Arg} / \mathrm{Arg}$ genotype tended to be associated with younger age of B-CLL manifestations and Pro/Pro with older age $(\mathrm{P}=0.04)$. But in contrast with Sturm et al., (2005), Kochethu et al., (2006) and Lahiri et al., (2007) who found that there are no significant differences in TP53 72 Arg/Pro genotype with means Age. This may be due to a variation in genotype frequencies. (See Table S2 )

Out of 110 cases, $79(71.8 \%)$ were males and 31 $(28.2 \%)$ were females $(\mathrm{M}: \mathrm{F}=2.6: 1)(\mathrm{P}=0.000)$. we found that there is no association between TP53 $72 \mathrm{Arg} /$ Pro polymorphisms and gender $(\mathrm{P}=0.358)$. Our result was consistent with those reported by Sturm et al., (2005), Kochethu et al., (2006), Lahiri et al., (2007) and Dong et al., (2014).

Depending on Binet stage system, forty-two (38.2\%) of patients presented in Binet stage $\mathrm{C}$, followed by thirty-five $(31.8 \%)$ presented at $\mathrm{B}$ stage and the rest thirty-three $(30.0 \%)$ at stage $\mathrm{A}$. We found that there is no significant association between TP53 72 Arg/Pro polymorphisms and Binet stages of our patients. Our result was consistent with previous studies done by Sturm et al., (2005), Kochethu et al., (2006), Lahiri et al., (2007) and Dong et al., (2014) who used Binet stage system to classify their CLL patients. On the other hand, depending on Rai stage system, we found that there was no significant association between TP53 72 Arg/Pro polymorphisms and Rai stages. No study found on literature reviewing discussed the association between TP53 72 Arg/Pro polymorphism and Rai stages system in CLL patients, this may be due to using of Binet system in European and American studies which used Rai system didn't discuss such association.

By using 30\% cutoff for positivity of CD38 expression,
$41 / 110(37.3 \%)$ of CLL patients were positive, and we did not find any association between TP53 $72 \mathrm{Arg} /$ Pro polymorphisms and $C D 38$ expression $(\mathrm{P}=0.782)$. However, our result agree with Sturm et al., (2005), Lahiri et al., (2007) and Dong et al., (2014), whereas disagree with a study conducted by Kochethu et al., (2006) found a weak association between Arg/Arg homozygous and CD38 negativity ( $\mathrm{P}=0.049)$, (See Table 2 ). Furthermore, there was no significant difference found in TP53 72 Arg/Pro genotype distribution in relation to $Z A P 70$ expression. Our result was consistent with all previous studies (Kochethu et al., 2006; Lahiri et al., 2007; Dong et al., 2014). There is no significant association was found, between TP53 72 Arg/Pro genotype and hematological parameters. These data are consistent with those previously study conducted in Sudan by (Wafaa Salah Aldeen Khogaly, 2015) and the only exception was with Haematocrit (See Table 3).

Limitations which are worth to mention are: sampling method was depended on voluntary participation, patients were not followed up for progression of B-CLL, survival rates and response to treatment administered after diagnosis confirmation and small size our control group unable to cover the differences ethnicity among Sudanese population, also not investigate the combined effects of TP53 mutation and this polymorphism in our population.

In conclusion our results can suggest that Pro/ Pro genotype contribute to increased susceptibility to B-Chronic Lymphocytic Leukemia risk in our population tenfold higher than those had Arg/Arg genotype, and Pro/ Pro genotype tend to be associated with older age patients, while no association was found between TP53 $72 \mathrm{Arg} /$ Pro and gender, clinical presentation, $C D 38$ expression, $Z A P 70$, and hematological parameters.

\section{Authors' contributions}

AAB, EAA and IKI conceived the study design, participated in data collection, performed the statistical analysis, interpreted the results, and revised the manuscript. AS, EAB, SOA and NHE participated in the statistical analysis and drafted the manuscript. OAA and EAF participated in the data collection, carried out the laboratory work, and prepared the results. $\mathrm{AAB}$ performed the molecular analysis. All authors read and approved the final manuscript.

\section{Ethics approval and consent to participate}

Ethical clearance was obtained from the Institutional Review Board at Al Neelain University. Principal investigator obtained written informed consent from all participants prior to their inclusion in the study.

\section{Competing interests}

The authors declare that they have no competing interests.

\section{Acknowledgments}

We would like to thank the staff of Haematology Department at Al Neelain University for facilities and support and we are grateful to the staff of Flowcytometry Laboratory for their collaboration. Finally, special thanks 
to the patients for being cooperative, despite their pain.

\section{References}

Bereir R, Mohamed H, Seielstad M, et al (2003). Allele frequency and genotype distribution of polymorphisms within disease-related genes is influenced by ethnic population sub-structuring in Sudan. Genetica, 119, 57-63.

Bilous N, Abramenko I, Chumak A, et al (2014). TP53 codon 72 single nucleotide polymorphism in chronic lymphocytic leukemia. Exp Oncol, 10, 258-61.

Binet J, Auquier A, Dighiero G, et al (1981). A new prognostic classification of chronic lymphocytic leukemia derived from a multivariate survival analysis. Cancer, 48, 198-206.

Chiorazzi N, Rai KR, Ferrarini M (2005). Chronic lymphocytic leukemia. N Engl J Med, 352, 804-15.

Cordone I, Masi S, Mauro FR, et al (1998). p53 expression in B-cell chronic lymphocytic leukemia: a marker of disease progression and poor prognosis. Blood, 91, 4342-9.

Dohner H, Fischer K, Bentz M, et al (1995). p53 gene deletion predicts for poor survival and non-response to therapy with purine analogs in chronic B-cell leukemias. Blood, 85, 1580-9.

Dong H-J, Fang C, Wang L, et al (2014). TP53 Pro72 allele potentially increases the poor prognostic significance of TP53 mutation in chronic lymphocytic leukemia. Med Oncol, 31, 908.

Dumont P, Leu J-J, Della Pietra AC, et al (2003). The codon 72 polymorphic variants of $\mathrm{p} 53$ have markedly different apoptotic potential. Nat Genet, 33, 357.

Hainaut P, Wiman KG (2009). 30 years and a long way into p53 research. Lancet Oncol, 10, 913-9.

Hallek M (2017). Chronic lymphocytic leukemia: 2017 update on diagnosis, risk stratification, and treatment. Am J Hematol, 92, 946-65.

Hallek M, Cheson BD, Catovsky D, et al (2008). Guidelines for the diagnosis and treatment of chronic lymphocytic leukemia: a report from the International Workshop on Chronic Lymphocytic Leukemia updating the National Cancer Institute-Working Group 1996 guidelines. Blood, 111, 5446-56.

Isobe M, Emanuel B, Givol D, et al (1986). Localization of gene for human $\mathrm{p} 53$ tumour antigen to band $17 \mathrm{p} 13$. Nature, 320, 84-5.

Kerr J, Al-Khattaf A, Barson A, et al (2000). An association between sudden infant death syndrome (SIDS) andHelicobacter pylori infection. Arch Dis Child, 83, 429-34.

Kochethu G, Delgado J, Pepper C, et al (2006). Two germ line polymorphisms of the tumour suppressor gene p53 may influence the biology of chronic lymphocytic leukaemia. Leuk Res, 30, 1113-8.

Lahiri O, Harris S, Packham G, et al (2007). p53 pathway gene single nucleotide polymorphisms and chronic lymphocytic leukemia. Cancer Genet Cytogenet, 179, 36-44.

Maciejewski JP, Tiu RV, O'Keefe C (2009). Application of array-based whole genome scanning technologies as a cytogenetic tool in haematological malignancies. $\mathrm{Br} J$ Haematol, 146, 479-88.

Majid A, Richards T, Dusanjh P, et al (2011). TP53 codon 72 polymorphism in patients with chronic lymphocytic leukaemia: identification of a subgroup with mutated IGHV genes and poor clinical outcome. Br J Haematol, 153, 533-5.

Mohand Altayeb Mohamed A, leena Babiker M, Ali EW (2013). Genotyping of P53 Exon 4 codon 72 in Sudanese patients with lymphoid leukemias. Int J Curr Res, 5, 3980-3.

Moreau EJ, Matutes E, A'hern RP, et al (1997). Improvement of the chronic lymphocytic leukemia scoring system with the monoclonal antibody SN8 (CD79b). Am J Clin Pathol, 108, 378-82.

Olivier M, Eeles R, Hollstein M, et al (2002). The IARC TP53 database: new online mutation analysis and recommendations to users. Hum Mut, 19, 607-14.

Oscier D, Dearden C, Eren E, et al (2012). Guidelines on the diagnosis, investigation and management of chronic lymphocytic leukaemia. Br J Haematol, 159, 541-64.

Pim D, Banks L (2004). p53 polymorphic variants at codon 72 exert different effects on cell cycle progression. Int $J$ Cancer, 108, 196-9.

Rai KR, Sawitsky A, Cronkite EP, et al (1975). Clinical staging of chronic lymphocytic leukemia. Blood, 46, 219-34.

Siddique M, Sabapathy K (2006). Trp53-dependent DNA-repair is affected by the codon 72 polymorphism. Oncogene, 25, 3489.

Själander A, Birgander R, Kivelä A, et al (1995). p53 polymorphisms and haplotypes in different ethnic groups. Hum Hered, 45, 144-9.

Sturm I, Bosanquet AG, Hummel M, et al (2005). In B-CLL, the codon 72 polymorphic variants of $\mathrm{p} 53$ are not related to drug resistance and disease prognosis. BMC Cancer, 5, 105.

Thomas M, Kalita A, Labrecque S, et al (1999). Two polymorphic variants of wild-type p53 differ biochemically and biologically. Mol Cell Biol, 19, 1092-100.

Tian X, Dai S, Sun J, et al (2016). Association between TP53 Arg72Pro polymorphism and leukemia risk: a meta-analysis of 14 case-control studies. Sci Rep, 6, 24097.

Wafaa Salah Aldeen Khogaly ME-FME-D, Eldirdiri Mohamed A, Enaam Abdelrhman A, Elshazali Widaa A (2015). Hematological findings in Sudanese patients with lymphoid leukemias with relation to P53 Arginine/proline polymorphism. Int J Info Res Rev, 2, 1192-4.

Weng Y, Lu L, Yuan G, et al (2012). p53 codon 72 polymorphism and hematological cancer risk: an update meta-analysis. PLoS One, 7, e45820.

Whibley C, Pharoah PD, Hollstein M(2009). p53 polymorphisms: cancer implications. Nat Rev Cancer, 9, 95.

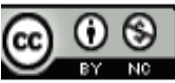

This work is licensed under a Creative Commons AttributionNon Commercial 4.0 International License. 